\title{
Dental Prosthetic Rehabilitation of a Tumor Patient After Radiotherapy and Mandibular Resection
}

\author{
Soo Jeong Portscher Kim*, Hans Christoph Lauer and Silvia Brandt \\ Department Dental, Oral and Orthodontic Medicine, Germany
}

*Corresponding author: Soo Jeong Portscher Kim, Department Dental, Oral and Orthodontic Medicine, Germany

\begin{abstract}
Background: Many patients with a history of head-and-neck tumors present with hard- and soft-tissue defects resulting from their disease and treatment that will preclude any dental prosthetic rehabilitation without the use of implants.

Case Report: A 57-year-old male patient with a history of tonsil and piriform sinus carcinoma sought prosthetic rehabilitation after successful radio-/chemotherapy and local mandibular resection. His dentition was severely reduced, consisting of inadequate crown restorations in the maxillary anterior segment and the mandibular right canine. The treatment decision was to rehabilitate the maxilla conventionally and the locally resected mandible with an implant-supported telescopic denture after extraction of tooth 43. Four implants were placed $(32,33,42,43)$ and allowed to osseointegrate submerged for 3 months, followed by surgical exposure, tightening abutments, and cementing conical crowns that served as primary telescopes. The secondary telescopes consisted of electroplated copings which were intraorally bonded to the tertiary structure, thus ensuring a perfect fit of the denture.

Conclusion: Other options for denture retention were contraindicated: ball or Locator attachments because of the existing xerostomia and pain from the resection-related scar tissue, and a bar due to its higher demands on self-performed oral hygiene and very high requirements for extending the superstructure in the event of an implant loss. The telescopic denture we provided has none of these drawbacks, nor does it require any extensive cantilever saddles because the masticatory loads are largely absorbed by the implants with almost no force transmission to the mucosa. The only treatment-related complication was a bony dehiscence, which was successfully managed by slightly reducing the bone and harvesting two connective-tissue grafts from the palate to close the perforation. After one year, the denture continues to be intact and functional. The patient reported a short period of adaptation followed by excellently restored function and greatly improved phonetics.
\end{abstract}

Keywords: head-and-neck tumors; radiotherapy; mandibular resection; prosthetic rehabilitation; telescopic denture

\section{Background}

Due to the sequelae of head-and-neck tumors, any dental prosthetic rehabilitation of patients with this disease remains a challenge [1,2]. Many have undergone chemo - and radiotherapy associated with surgical removal of affected tissue with a healthy margin $[3,4]$. Such resection will leave behind hard- and softtissue defects which, depending on the severity of the case, can be addressed either by plastic surgery coverage or by prosthetic reconstruction [5]. The latter route is taken to compensate for the incurred losses in function, phonetics and esthetics because all of these deficits are known to compromise the patients' quality of life [6]. Additional challenges to treatment arise from irradiationinduced caries and xerostomia [7,8]. In most cases, this situation will be further compounded by a dentition so severely reduced that additional implants will be required to serve as abutments for an optimal prosthetic solution [9]. In this article, we report a treatment case of this kind which, due to the aforementioned problems, did not allow for a conventional mucosa-supported prosthetic solution.

\section{Clinical Case}

A 57-year-old male patient with a recent history of tonsil carcinoma and secondary carcinoma of the piriform sinus presented for prosthetic consultation in our outpatient unit (Johann-Wolfgang-Goethe University, Frankfurt am Main, Germany). The tumors had been successfully treated by combined radio- and chemotherapy, also including mandibular resection in the anterior segment (sites 43 to 41 ). Also noteworthy about the 
patient's medical history is the fact that he continues to smoke even today, with a self-reported tobacco use of around 10 cigarettes a day. His dental history was such that the oral examination revealed inadequate crown restorations in the maxillary anterior segment (13 to 23) and the right canine as the only residual tooth in the mandible (43). Radiographic and photographic views of the initial situation are provided in (Figures 1-3). Not wearing a denture, the patient requested a prosthetic therapy to restore function. He also complained about phonetic deficits, feeling that his edentulism rendered him unable to participate in public life and that this was a major psychological burden.

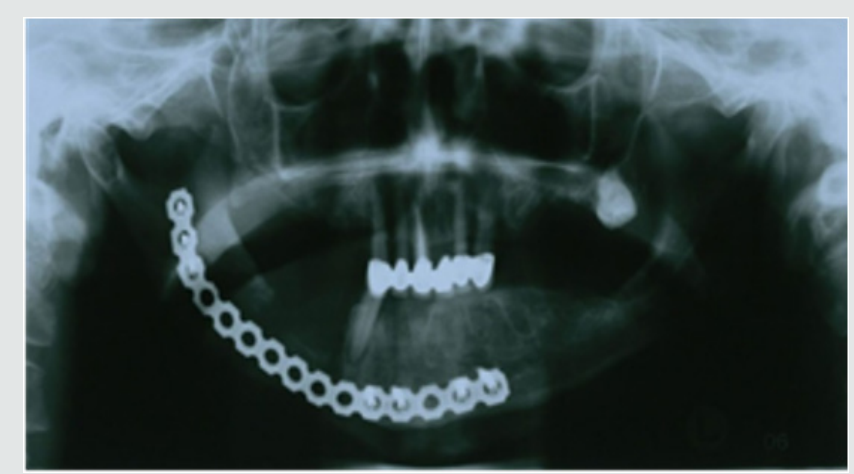

Figure 1: Orthopantomogram.

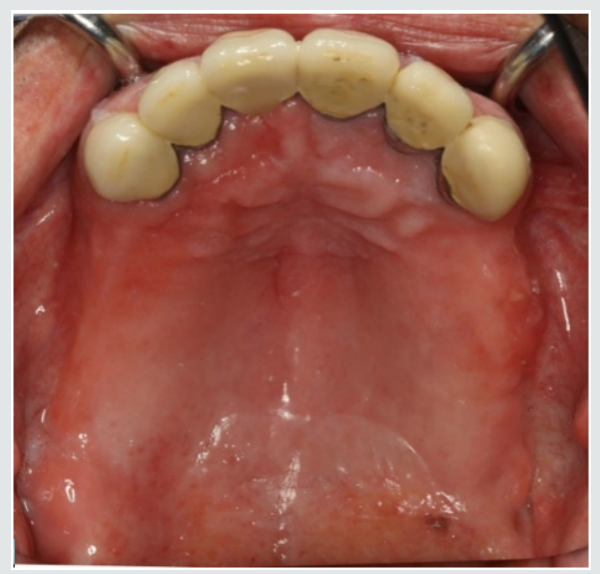

Figure 2: Initial situation of the maxilla.

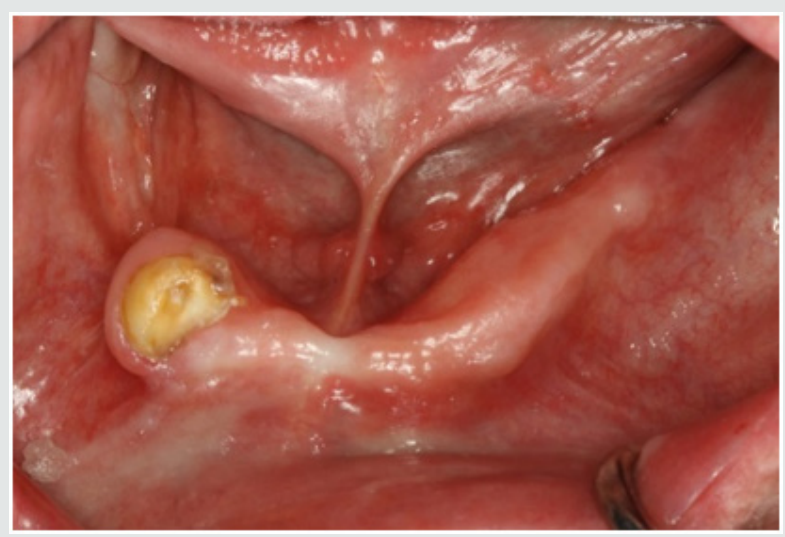

Figure 3: Initial situation of the mandible.
After discussing all prosthetic options, he decided to save money in the maxilla by settling for a conventional solution with a single crown for the right lateral incisor (12), a three-unit fixed partial denture from the right central to the left lateral incisor (11 to 22), and a one-piece cast denture including conical crowns on both canines (13 and 23). In the mandible with its hard-tissue defect and scar tissue, he opted for an implant-supported telescopic denture with conical primary crowns and electroplated secondary copings. The first aim of treatment was to restore the patient to correct occlusal relations after his longstanding edentulism. This was accomplished by fabricating interim dentures for the maxilla and mandible, to be worn for a 3-month test period (Figure 4). The patient had no problem readjusting to the new vertical dimension of occlusion, and neither did he exhibit any temporomandibular joint symptoms. The next step was to extract tooth 43 and to insert four implants $9 \mathrm{~mm}$ in length (Astra $\mathrm{Tech}^{\mathrm{TM}}$ Osseo Speed ${ }^{\mathrm{TM}} \mathrm{TX}$; Dentsply Sirona, Mannheim, Germany) in the lateral incisor and canine positions of the mandible $(32,33,42,43)$.

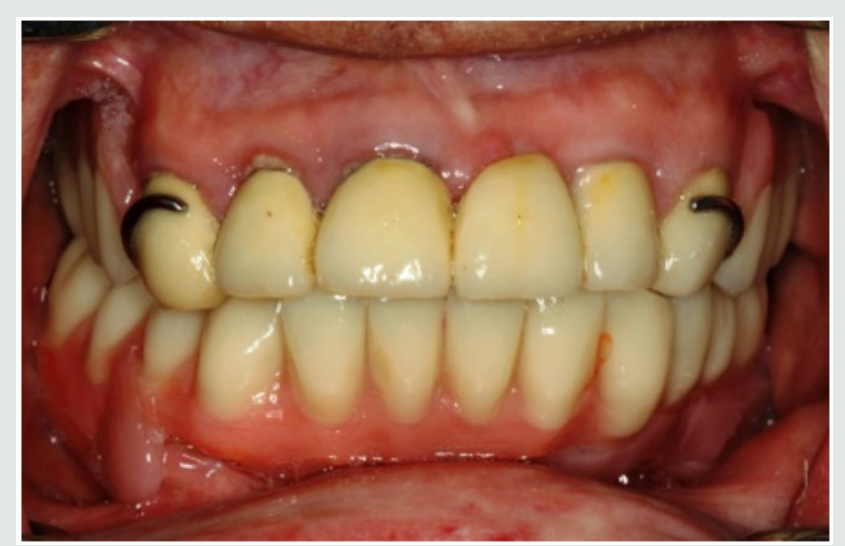

Figure 4: Interim dentures of the maxilla and mandible.

The ensuing 3-month period of submerged osseointegration was utilized to implement the afore mentioned plan of maxillary prosthetic rehabilitation. To this end, the inadequate crowns in the maxillary anterior segment (13 to 23 ) were removed and the residual tooth structures prepared, followed by taking a precision impression in polyether material (Impregum $^{\mathrm{TM}}$ Penta $^{\mathrm{TM}}$; 3M ESPE, Seefeld, Germany). The next session was dedicated to determining the horizontal and vertical interarch relationships, followed by an arbitrary facebow transfer and mounting of the casts (SAM 2P; SAM Präzisionstechnik, Gauting, Germany). A first firing and intraoral try-in of the resultant crowns took place to implement any modifications dictated by the patient's esthetic expectations. Minor adjustments to the tooth shapes were needed for finalization. The next session was dedicated to final cementation $\left(\mathrm{Ketac}^{\mathrm{TM}} \mathrm{Cem} ; 3 \mathrm{M}\right.$ ESPE) of the single crown (12), the fixed partial denture (11 to 22 ), as well as the two conical crowns in the canine positions for subsequent insertion of the denture $(13,23)$.

In the mandible, the 3-month osseointegration period was followed by a second-stage procedure to expose the implants. The 
prosthetic treatment phase was delayed by a bony dehiscence (32 to 36 ), which was surgically induced but successfully managed by slightly reducing the local bone and harvesting two connectivetissue grafts from the palate to close the perforation (Figures 5 \& 6). Once the graft had healed with no further dehiscence, we proceeded to take an open-tray impression (Figure 7) of the implants in polyether (Impregum ${ }^{\mathrm{TM}}$ Penta $^{\mathrm{TM}} ; 3 \mathrm{M}$ ESPE). As the upper restorations were already in situ, another impression was taken of the maxilla in alginate, followed by an arbitrary facebow transfer (SAM 2P; SAM Präzisionstechnik). Given the difficult implant positions, the interarch relationships were determined horizontally and vertically, followed by an esthetic try-in implemented as a mockup in wax to optimize together with the patient the parameters of tooth shape, shade and position (Figures $8 \& 9$ ).

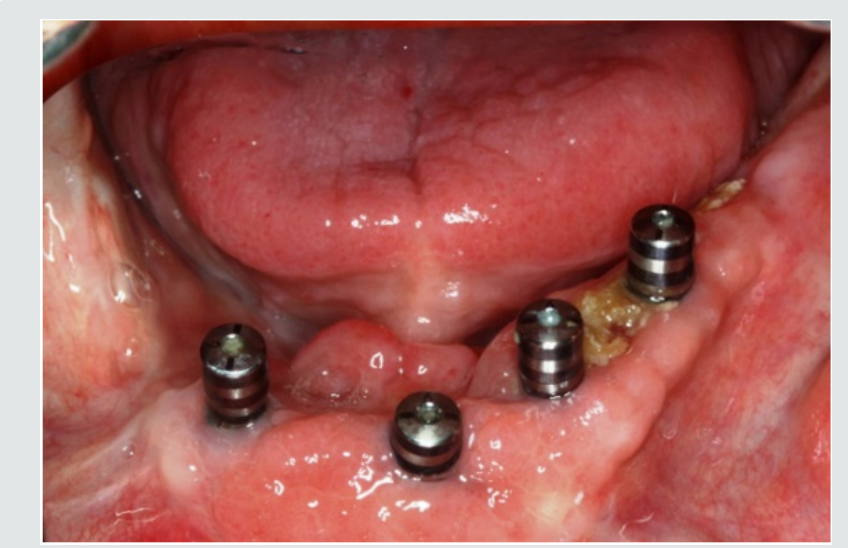

Figure 5: Bony dehiscence in the third quadrant.

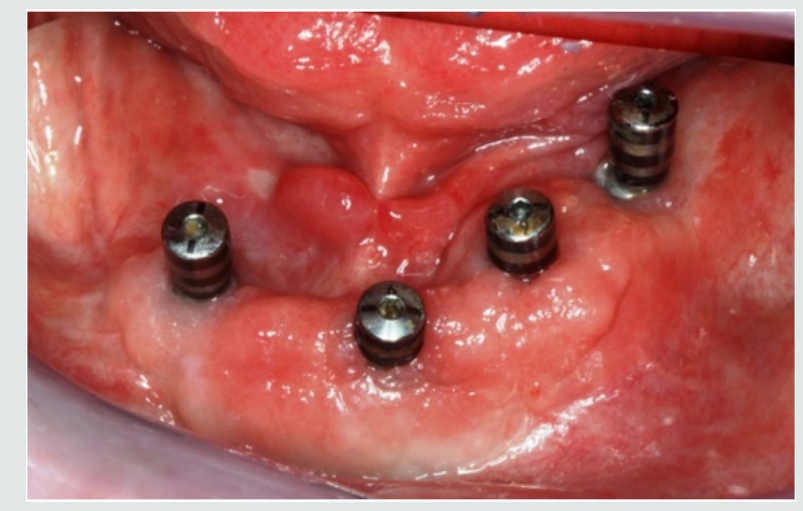

Figure 6: Situation after covering the dehiscence.

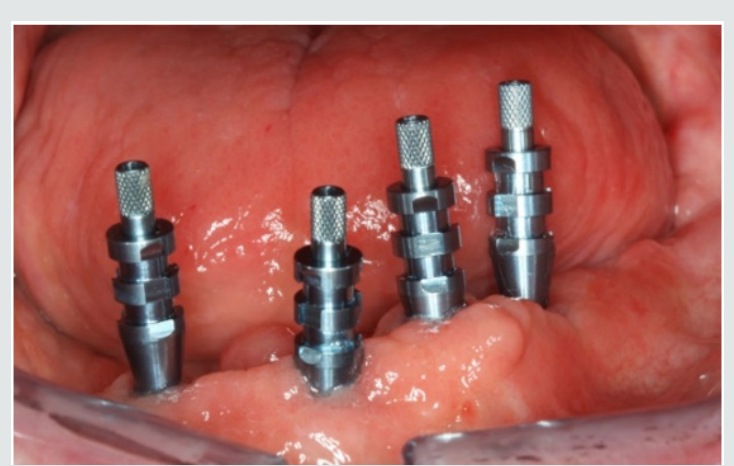

Figure 7: Impression posts to receive an open tray.

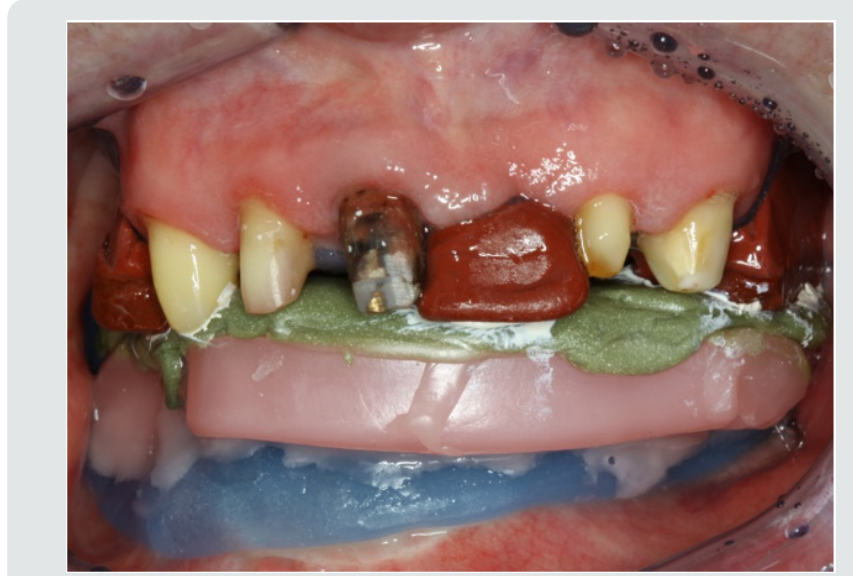

Figure 8: Determining the interarch relationships.

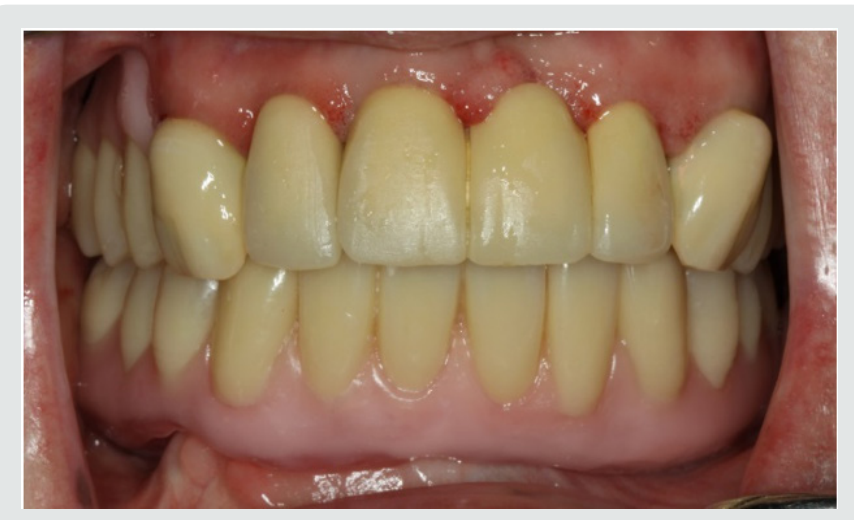

Figure 9: Esthetic mock-up.

During the next session, the primary conical crowns for the telescopic denture were definitively inserted. The first step was to tighten implant abutments to the implant fixtures (with the aid of a transfer index) and to seal the screw holes with a foam pellet and blue wax. Then a try-in of the primary ceramic crowns was performed, with the electroplated secondary copings and the tertiary framework placed on top, followed by definitive cementation of the primary crowns with $\mathrm{Ketac}^{\mathrm{TM}}$ Cem (3M ESPE). After doublechecking the framework for passive seating, a self-curing adhesive (Nimetic $^{\mathrm{TM}}$ Cem, 3M Espe) was used for intraoral bonding of the secondary copings to the tertiary framework, thus ensuring a perfect fit of the superstructure. This framework assembly was now used to determine the final interarch relationships and was indexed with self-curing resin (Pro Temp ${ }^{\mathrm{TM}}, 3 \mathrm{M}$ ESPE), followed by an over impression of the framework that included indexing with a polyether material (Impregum ${ }^{\mathrm{TM}}$ Penta $^{\mathrm{TM}}$; 3M ESPE).

With the conical crowns serving as primary telescopes definitively in situ at this point, a laboratory-made "travel denture" could immediately be provided that featured soft relining with a silicone material (Mollosil ${ }^{\circledR}$ Plus; Detax, Ettlingen, Germany). The main purpose of this restoration is to protect the primary crowns, but it also allows patients to be discharged with a well-fitting denture at an early stage and, in addition, can later be used as a substitute whenever the final denture may need to be repaired. The next session was used for another wax mock-up, whereupon 
the telescope denture could be completed and delivered (Figures 10-12). Due to the precise fit of the framework, the denture base could be designed as delicately as possible just as required by the intraoral situation and with no need to involve the reflection fold or the retromolar triangle. At the 12-month recall, the denture was intact and functional. The patient reported that, after a brief period of adaptation, his function had been excellent and his phonetics greatly improved, so that he once again felt comfortable participating in public life.

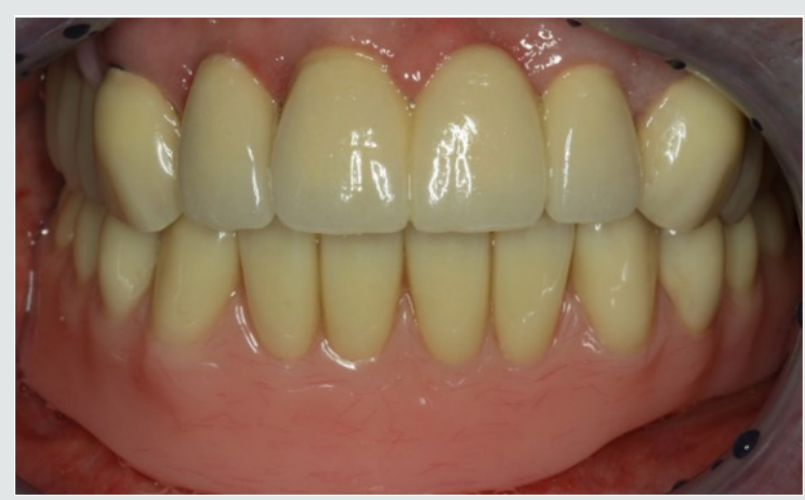

Figure 10: Intraoral delivery.

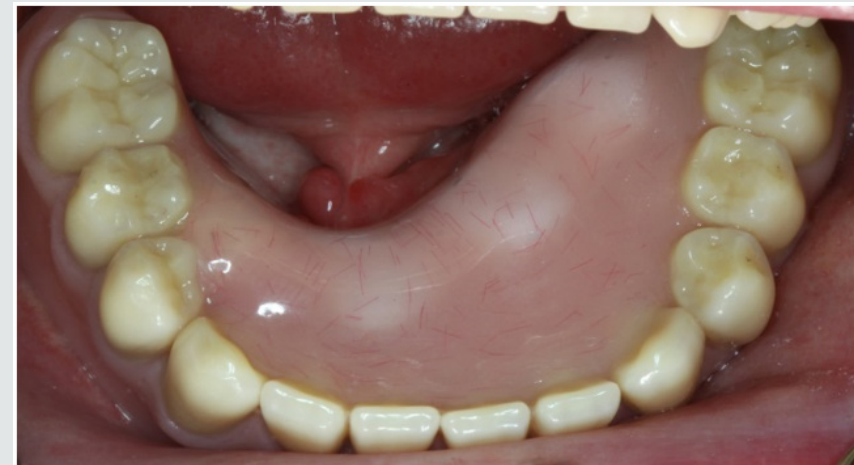

Figure 11: Occlusal view of the mandibular telescopic denture.

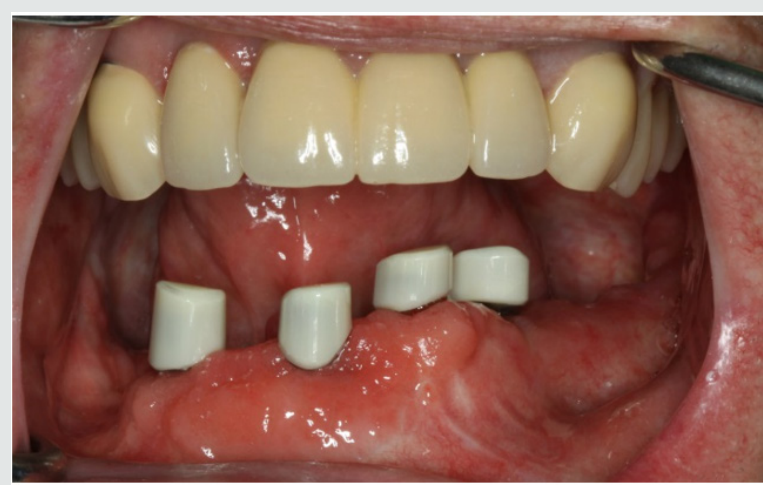

Figure 12: Anterior view of the ceramic primary crowns.

\section{Discussion}

Many patients with a history of head-and-neck tumor will mandatorily require implants for prosthetic rehabilitation. One to four implants are needed to adequately retain a removable superstructure in the edentulous mandible. Ball attachments necessitate at least one implant [10] and Locator attachments at least two implants [11], so that one or two implants may suffice to offer adequate retention [12]. At the same time, these prefabricated attachments have limitations that adversely influence their positional stability and retention and, because of this low retention, require a larger surface area. Both attachment types would have been contraindicated in the case here discussed, considering that the base of such a denture would irritate the mucosa and cause pain due to the existing xerostomia and the local scar tissue, respectively. To avoid these problems, a superstructure on four implants was required. Eligible modes of retention in this context would be telescopic crowns or a bar structure [13].

A bar offers good retention and stability, but its splinting of the implants would have imposed significant demands on the patient's ability to perform oral hygiene. In addition, the requirements for extending the superstructure in the event of an implant loss would be very high. Hence it was decided to restore the patient's mandible with a telescopic denture. Our concept of implementing the telescopes as conical primary crowns and electroplated secondary copings has several advantages. It offers high retention, simple insertion and removal, as well as optimal conditions for hygiene because the patient can freely access all surface areas thanks to the fact that each implant is fitted with an independent primary crown $[14,15]$. Also, the superstructure can be readily extended if required, thus ensuring continued function should an implant be lost. Lastly, these telescopic dentures do not require any extensive cantilever saddles because the masticatory loads are largely absorbed by the implants with almost no force transmission to the mucosa.

\section{References}

1. Schoen PJ, Reintsema H, Raghoebar GM, Vissink A, Roodenburg JL (2004) The use of implant retained mandibular prostheses in the oral rehabilitation of head and neck cancer patients. A review and rationale for treatment planning. Oral Oncol 40(9): 862-871.

2. Butterworth C, McCaul L, Barclay C (2016) Restorative dentistry and oral rehabilitation: United Kingdom National Multidisciplinary Guidelines. J Laryngol Otol 130(S2): S41-S44.

3. Grötz KA, Krummenauer F, al Nawas B, Kuffner HD, Reichert TE, et al. (2000) Retrospective analysis of the prognostic significance of the manner of mandibular resection and adjuvant radiotherapy in paramandibular carcinomas. Mund Kiefer Gesichtschir 4(5): 301-308.

4. (2002) Grötz.

5. Rissa E, Kurki T, Laine J, Närhi T (2018) Prosthetic rehabilitation with implant-supported mandibular overdenture after partial glossectomy and radiation therapy: a case report. Eur J Prosthodont Restor Dent 26(2): 95-99.

6. Heydecke G, Boudrias P, Awad MA, De Albuquerque RF, Lund JP, et al. (2003) Within-subject comparisons of maxillary fixed and removable implant prostheses: patient satisfaction and choice of prosthesis. Clin Oral Implants Res 141(1): 125-130.

7. Ngeow WC, Chai WL, Rahman RA, Ramli R (2006) Managing complications of radiation therapy in head and neck cancer patients: Part I. Management of xerostomia. Singapore Dent J 28(1): 1-3. 
8. Chai WL, Ngeow WC, Ramli R, Rahman RA (2006) Managing complications of radiation therapy in head and neck cancer patients: Part II. Management of radiation-induced caries. Singapore Dent J 28(1) 4-6.

9. Hong CH, Napeñas JJ, Hodgson BD, Stokman MA, Mathers-Stauffer V, et al. (2010) A systematic review of dental disease in patients undergoing cancer therapy. Support Care Cancer 18(8): 1007-1021.

10. Passia N, Brezavšcek M, Fritzer E, Kappel S, Kern T, et al. (2014) Single dental implant retained mandibular complete dentures--influence of the loading protocol: study protocol for a randomized controlled trial. Trials 15: 186 .

11. Guédat C, Nagy U, Schimmel M, Müller F, Srinivasan M (2018) Clinical performance of LOCATOR ${ }^{\circledR}$ attachments: a retrospective study with 1-8 years of follow-up. Clin Exp Dent Res 4(4): 132-145.
12. Cakarer S, Can T, Yaltirik M, Keskin C (2011) Complications associated with the ball, bar and Locator attachments for implant-supported overdentures. Med Oral Patol Oral Cir Bucal 16: e953-e959.

13. Eitner S, Schlegel A, Emeka N, Holst S, Will J, et al. (2008) Comparing bar and double-crown attachments in implant-retained prosthetic reconstruction: a follow-up investigation. Clin Oral Implants Res 19(5): 530-537.

14. Rinke S, Buergers R, Ziebolz D, Roediger M (2015) Clinical outcome of double crown-retained implant overdentures with zirconia primary crowns. J Adv Prosthodont 7(4): 329-337.

15. Weigl P, Hahn L, Lauer HC (2000) Advanced biomaterials used for a new telescopic retainer for removable dentures. J Biomed Mater Res 53(4): 320-336.

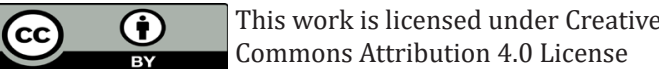

To Submit Your Article Click Here:

Submit Article

DOI: $10.32474 /$ SCSOAJ.2019.02.000129

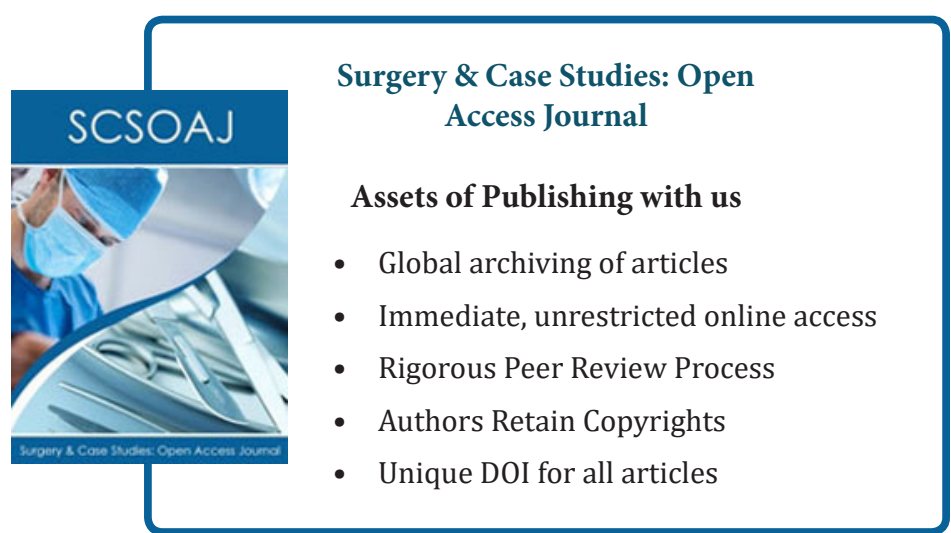

